\title{
Risk factors associated with pulmonary exacerbations in pediatric patients with cystic fibrosis
}

\author{
Silvina Lubovich, M.D. ${ }^{a}$, Silvina Zaragoza, M.D. ${ }^{a}$, Viviana Rodríguez, M.D. ${ }^{a}$, \\ Jefferson Buendia, M.D. ${ }^{b}$, Bethy Camargo Vargas, M.D. ${ }^{a}$, \\ Jessica Alchundia Moreira, M.D. ${ }^{a}$, Laura Galanternik, Bacteriologistc, \\ Patricia Ratto, Kinesiologist ${ }^{d}$ and Alejandro Teper, M.D. ${ }^{a}$
}

\begin{abstract}
Introduction. Cystic fibrosis patients develop pulmonary exacerbations (PEs) that require intravenous treatment. Theobjective of this study was to determine the risk factors associated with PEs and establish the percentage of patients who failed to recover their lung function.
\end{abstract}

Population and methods. Observational, retrospective, cohort study. The medical records of cystic fibrosis patients seen at Hospital de Niños Ricardo Gutiérrez in 2013 were reviewed. Patients were divided into group 1, with PE (Fuchs criteria), and group 2, without PE. Age, sex, p.F508del mutation, percentage of baseline forced expiratory volume in the first second, baseline body mass index Z-score, chronic Pseudomonas aeruginosa, methicillin-resistant Staphylococcus aureus and Burkholderia cepacia complex colonization (Leeds criteria), percentage of cystic fibrosis-related diabetes, and recovery of baseline forced expiratory volume in the first second were recorded.

Results. A total of 117 patients were included. Group 1: 50, group 2: 67 patients. PEs were associated with a lower body mass index Z-score (RR: $1.45 ; p=0.002)$, p.F508del mutation (RR: 3.23; $p=0.05)$, and chronic Burkholderia cepacia complex (RR: $3.69 ; p=0.002$ ), Pseudomonas aeruginosa (RR: $1.89 ; p=0.01)$ and methicillinresistant Staphylococcus aureus colonization (RR: $2.32 ; p=0.002)$. Twenty-four percent of patients failed to recover their lung function.

Conclusions. The presence of the p.F508del mutation, a poor nutritional status, and chronic colonization were the risk factors for exacerbation. A fourth of patients failed to recover their lung function.

Key words: pulmonary exacerbations, cystic fibrosis.

http: / / dx.doi.org/10.5546/ aap.2019.eng.e466

To cite: Lubovich S, Zaragoza S, Rodríguez V, Buendía J, et al. Risk factors associated with pulmonary exacerbations in pediatric patients with cystic fibrosis. Arch Argent Pediatr 2019;117(5):e466-e472.
E-mail address:

Silvina Lubovich, M.D.: slubovich@gmail.com

Funding:

None.

Conflict of interest:

None.

Received: 9-8-2018

Accepted: 2-25-2019

\section{INTRODUCTION}

Cystic fibrosis (CF) is an inherited, autosomal, recessive genetic disorder characterized by an altered exocrine secretion. CF is a chronic, progressive disease with multisystem involvement, caused by mutations in a protein called the cystic fibrosis transmembrane conductance regulator (CFTR).

The natural history of CF includes a progressive deterioration of lung function, with episodes of respiratory symptom worsening called "pulmonary exacerbations". ${ }^{1,2}$

In spite of the remarkable improvement in lung function and nutritional status in recent years, no substantial changes have been observed in the frequency of pulmonary exacerbations. ${ }^{3}$ According to the United States Cystic Fibrosis Patient Registry, in 2013, the percentage of exacerbations requiring intravenous antibiotics for the entire CF population was $35 \%$ while, in 2016, it was $33.8 \%$.

Patients aged $15-30$ years have the greater probability of having an exacerbation. ${ }^{3}$ There is no universal definition of pulmonary exacerbation, but the most typical symptoms include increased cough or sputum production, fever, weight loss, reduced exercise tolerance, and new clinical findings, such as tachypnea, new rales on auscultation, reduced lung function, decreased saturation or new radiographic findings. ${ }^{4}$ Bilton et al., ${ }^{5}$ have defined pulmonary exacerbation as the need to indicate an antibiotic therapy due to a recent change in clinical parameters. If an exacerbation is severe, it requires intravenous antibiotic therapy. 
Several publications have considered the need for intravenous antibiotic therapy in order to define an exacerbation. ${ }^{6,7}$ In this study, we based the definition of exacerbation on Fuchs criteria. ${ }^{8}$

Pulmonary exacerbations are associated with a greater deterioration in lung function and a reduced quality of life and survival. ${ }^{9-11}$ After a pulmonary exacerbation treated with intravenous antibiotics, up to $25 \%$ of patients fail to recover their lung function. ${ }^{12}$

Few studies have determined the risk factors for pulmonary exacerbations in CF. In a retrospective study, Schechter et al., ${ }^{13}$ observed that patients with a low socioeconomic level were more prone to developing pulmonary exacerbations. In a prospective study done in adult patients with multidrug-resistant bacterial infections, Block et al., ${ }^{14}$ determined that younger age, female sex, reduced baseline lung function, and a history of multiple pulmonary exacerbations were associated with having exacerbations. Knowing these risk factors would allow us to identify them in an early manner and provide a timely intervention that would prevent an exacerbation.

The objective of this study was to identify the risk factors associated with pulmonary exacerbations in children with $\mathrm{CF}$ and describe the percentage of patients who fail to recover their baseline lung function after an exacerbation.

\section{POPULATION AND METHODS}

This was an observational, analytical study conducted in a retrospective cohort. It was approved by the Ethics and Teaching Committee of Hospital de Niños Ricardo Gutiérrez.

\section{Population}

Inclusion criteria

- Patients younger than 18 years, diagnosed with CF (2 positive sweat tests or 2 mutations in the CFTR gene causing $C F$ ), who received follow-up by the Respiratory Center of Hospital de Niños Ricardo Gutiérrez in 2013 and who attended at least 4 control visits during the year.

\section{Exclusion criteria}

- Patients in the waiting list for a lung transplant.

- Patients who had a scheduled hospitalization.

\section{Procedures}

The medical records of all patients who met the inclusion criteria were reviewed, and collected data were anonymized. Pulmonary exacerbation was defined based on Fuchs criteria ${ }^{8}$ (hospitalization with parenteral antibiotics with the patient having 4 or more of the following 13 signs or symptoms: changes in sputum, hemoptysis, increased cough, increased dyspnea, malaise, fatigue/lethargy, fever, anorexia/ weight loss, sinus pain, increased sinus discharge, change in the exam of the chest, decreased lung function $\geq 10 \%$ or radiographic changes). The demographic, clinical, bacteriological, and functional characteristics of patients with $\mathrm{CF}$ who had at least a pulmonary exacerbation in 2013 and required hospitalization were recorded. These patients were included in group 1 (with exacerbation) and compared to CF patients who had attended control visits at the same center but had not required hospitalization due to an exacerbation in the same period, who were included in group 2 (without exacerbation). A total of 83 exacerbation episodes were documented. The need for oral antibiotics was not analyzed in any of the groups. The following clinical outcome measures were recorded: age, sex, baseline body mass index (BMI) Z-score in children older than 2 years (based on height and weight 3-6 months before the exacerbation during a stable condition and, in group 2, the best score in the same period), cystic fibrosisrelated diabetes (CFRD), presence of at least 1 p.F508del mutation. The percentage of patients with chronic methicillin-resistant Staphylococcus aureus (MRSA), Pseudomonas aeruginosa (PA) and Burkholderia cepacia complex (BCC) infection was also recorded. Patients were considered to have a chronic infection if they had more than $50 \%$ of positive cultures out of at least 4 cultures done in that year period (Leeds criteria). ${ }^{15}$ Bronchial secretions were obtained from an oropharyngeal swab in patients younger than 4 years, and from spontaneous sputum expectoration in older ones. Samples were seeded in blood agar, cystine lactose electrolyte deficient (CLED) agar, chocolate agar, cetrimide agar, mannitol salt agar, and Burkholderia cepacia Selective Agar (BCSA). Isolated microorganisms were identified using conventional biochemical tests.

Children 6 years and older had a spirometry done as per the American Thoracic Society (ATS) criteria. The percentage of predicted baseline forced expiratory volume in the first second 
$\left(\mathrm{FEV}_{1}\right.$ ) was recorded as per Knudson (who considered the best $\mathrm{FEV}_{1}$ value 3-6 months before the pulmonary exacerbation); in group 2 , the best $\mathrm{FEV}_{1}$ value in the same year period was used. $\mathrm{FEV}_{1}$ was also recorded at the beginning and end of the exacerbation. To obtain the percentage of patients who recovered their lung function after the exacerbation, the $\mathrm{FEV}_{1}$ on the last day of hospitalization was obtained and compared to the baseline $\mathrm{FEV}_{1}$ (3-6 months before the exacerbation). If the patient achieved $90 \%$ or more of his/ her baseline $\mathrm{FEV}_{1}$, lung function was considered recovered.

Antibiotic therapy duration was determined by a specialist in CF based on symptoms and lung function recovery.

All outcome measures were compared in both groups to establish associations between outcome measures and the frequency of exacerbations.

\section{Statistical analysis}

Categorical outcome measures were described as frequency and percentage, while numerical outcome measures were expressed as mean, standard deviation, and confidence intervals (CIs) if they had a normal distribution or as median and interquartile range if they had an abnormal distribution.

The Poisson regression model was used to measure the association between annual exacerbation rates and the different studied outcome measures. The backward technique was used, eliminating those outcome measures that did not reach a $p$ value below $20 \%$. The criterion for continuation in the model was a significance level of less than or equal to $5 \%$. All analyses were done using the STATA $11.0^{\circ}$ software.

For the sample size of 117 patients (n), a 0.39 odds ratio (OR) and a 0.20-0.55 $95 \% \mathrm{CI}$ were estimated, with a 0.59 event frequency in the exposed group, a 0.05 type I error, and a $90 \%$ power (1- type II error), according to the magnitude of the association observed among the different risk factors and the exacerbation reported in Block's study. ${ }^{14}$

\section{RESULTS}

Out of 125 patients under follow-up, 117 were included; their age ranged between 2 months and 18 years: 50 were included in the group with exacerbation and 67 , in the group without exacerbation. Two patients were excluded because they were in the waiting list for bipulmonary transplant; 4 , because they did not have at least 4 secretion cultures in that year; and 2, because they were older than 18 years. The groups are described in Table 1.

Out of 67 patients without exacerbation, $50(74.6 \%)$ showed the usual flora or methicillinsensitive Staphylococcus aureus (MSSA) in their bronchial secretions; none had chronic BCC colonization, and only 2 had MRSA and PA co-infection. In the group of 50 patients with exacerbation, only $5(10 \%)$ had the usual flora or MSSA, and $9(18 \%)$ had MRSA and PA coinfection.

In relation to lung function, out of 117 patients, a baseline spirometry was obtained in children

TABLE 1. Population of patients younger than 18 years and diagnosed with cystic fibrosis seen at Hospital de Niños Ricardo Gutiérrez in 2013

\begin{tabular}{|c|c|c|c|c|}
\hline & $\begin{array}{l}\text { Group 1: } \\
\text { with exacerbation } \\
(n=50)\end{array}$ & $\begin{array}{c}\text { Group 2: } \\
\text { without exacerbation } \\
(n=67)\end{array}$ & OR $(95 \%$ CI $)$ & $p$ \\
\hline \multicolumn{5}{|l|}{ Age in years } \\
\hline Mean (SD) & $10.03(6.37)$ & $7.73(4.43)$ & $1.07(1.01,1.14)$ & 0.030 \\
\hline Female sex $(\%)$ & $27(54)$ & $26(38)$ & $1.85(0.88,3.88)$ & 0.104 \\
\hline \multicolumn{5}{|l|}{ Baseline BMI Z-score } \\
\hline Mean (SD) & $-0.65(1.07)$ & $-049(0.97)$ & $1.81(1.17,2.8)$ & 0.007 \\
\hline p.F508del (\%) & $45(93)$ & $48(71)$ & $5.93(1.64,21.43)$ & 0.007 \\
\hline \multicolumn{4}{|l|}{ Baseline $\mathrm{FEV}_{1}$} & 0.000 \\
\hline CFRD $(\%)$ & $10(20)$ & $0(0)$ & NA & 0.000 \\
\hline $\mathrm{BCC}(\%)$ & $5(10)$ & $0(0)$ & NA & 0.008 \\
\hline $\mathrm{PA}(\%)$ & $20(40)$ & $8(11)$ & $4.91(1.93,12.46)$ & 0.001 \\
\hline MRSA (\%) & $20(40)$ & $9(13)$ & $4.29(1.74,10.58)$ & 0.002 \\
\hline
\end{tabular}

SD: standard deviation; BMI: body mass index; $\mathrm{FEV}_{1}$ : forced expiratory volume in the first second; CFRD: cystic fibrosis-related diabetes; BCC: Burkholderia cepacia complex; PA: Pseudomonas aeruginosa; MRSA: methicillin-resistant Staphylococcus aureus; NA: not available; OR: odds ratio; CI: confidence interval. 
aged 6 years and older, i.e., 38 patients from the group without exacerbation and 33 from that with exacerbation. The average baseline $\mathrm{FEV}_{1}$ (predicted percentage) was $102 \% \pm 15$ in the group without exacerbation and $68.9 \% \pm 20.28$, in that with exacerbation (OR: 0.88 ; $95 \%$ CI: 0.840.93; $p<0.0001$ ) (Figure 1).

None of the 38 patients without exacerbations had a baseline $\mathrm{FEV}_{1}$ below $60 \%$, while 10 out of the 33 patients with exacerbations $(30 \%)$ had a baseline $\mathrm{FEV}_{1}$ below $60 \%$ of the predictive percentage $(p<0.001)$.

When comparing both groups in the univariate analysis, significant differences were observed in terms of age, BMI Z-score, presence of CFRD, having at least 1 p.F508 mutation, the percentage of baseline $\mathrm{FEV}_{1}$, and having a chronic colonization by any of the 3 bacteria (Table 2). However, the multivariate analysis showed that the outcome measures that predicted exacerbations were a lower BMI Z-score, having at least 1 p.F508del mutation, and chronic BCC, PA or MRSA colonization (Table 3).

\section{Group with pulmonary exacerbations}

A total of 83 exacerbation episodes were recorded. The median length of hospitalization was 14 days (range: 13-36). In 16 exacerbation episodes (18.8\%), patients required non-invasive ventilation. No patient died during a pulmonary exacerbation.

Out of a total of 50 exacerbation episodes with available spirometry values, in 46 , patients had an improved $\mathrm{FEV}_{1}$ from baseline after antibiotic therapy (92\%). In addition, out of 33 patients with exacerbations with available baseline spirometry values, $8(24 \%)$ failed to recover their lung function (Figure 2).

TABLE 2. Univariate analysis

\begin{tabular}{lcc}
\hline & RR $(\mathbf{9 5} \% \mathbf{C I})$ & $\mathbf{p}$ \\
\hline Age & $1.04(1.01,1.08)$ & 0.024 \\
Female sex & $1.36(0.88,2.09)$ & 0.160 \\
p.F508del mutation & $6.07(1.91,19.24)$ & 0.002 \\
CFRD & $2.96(1.74,4.99)$ & 0.000 \\
Lower BMI Z-score & $1.69(1.35,2.13)$ & 0.000 \\
Baseline FEV1 & $0.96(0.95,0.97)$ & 0.000 \\
BCC & $2.38(1.15,4.95)$ & 0.019 \\
PA & $1.79(1.14,2.81)$ & 0.010 \\
MRSA & $2.44(1.58,3.76)$ & 0.000 \\
\hline
\end{tabular}

CFRD: cystic fibrosis-related diabetes; BMI: body mass index; $\mathrm{FEV}_{1}$ : forced expiratory volume in the first second; BCC: Burkholderia cepacia complex; PA: Pseudomonas aeruginosa; MRSA: methicillin-resistant Staphylococcus aureus; RR: relative risk; CI: confidence interval.

FIGURE 1. Baseline forced expiratory volume in the first second as a percentage of that predicted in patients with and without exacerbation

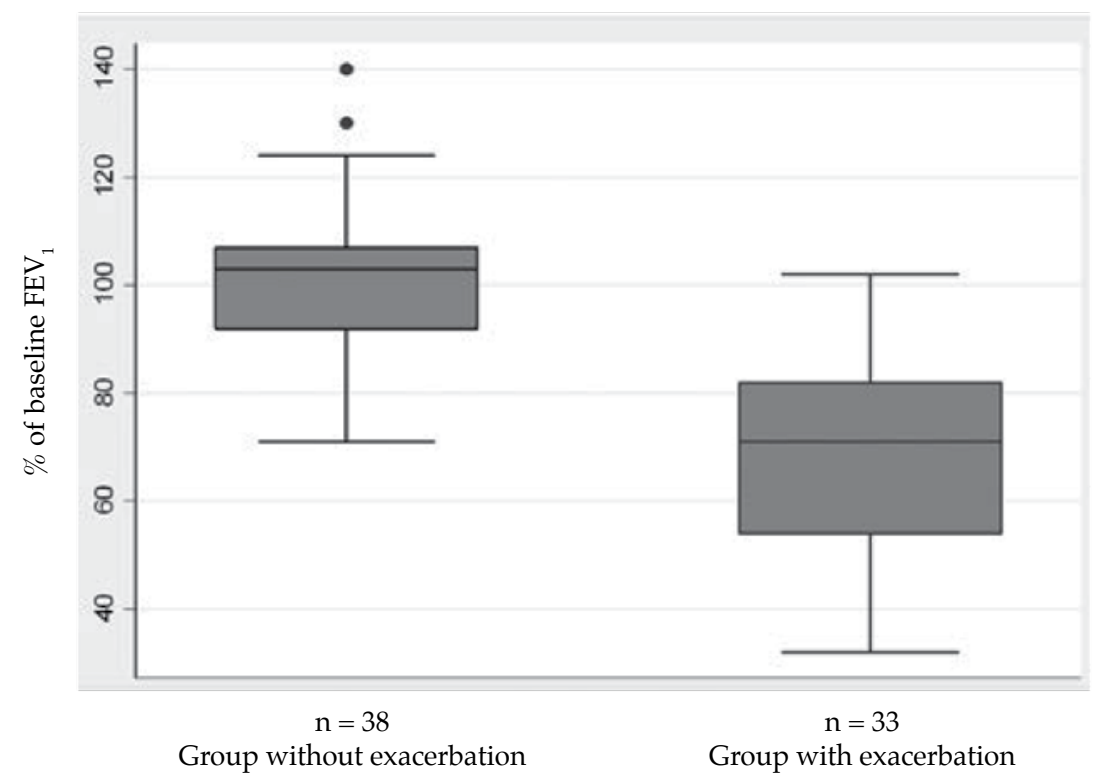

$\mathrm{FEV}_{1}$ : forced expiratory volume in the first second. 


\section{DISCUSSION}

Pulmonary exacerbations in CF have a clear impact on the quality of life of patients, and it is known that these results in an impaired lung function and contribute to diseaserelated morbidity and mortality. Liou et al., ${ }^{16}$ demonstrated, in 2001, that pulmonary exacerbations reduced patient survival by $12 \%$.

Schechter et al., ${ }^{13}$ found that a low socioeconomic level was a risk factor associated with having a pulmonary exacerbation in CF patients. In our study, this was not assessed as a risk factor because most of our patients had a low socioeconomic level.

Block et al., ${ }^{14}$ observed that female sex, younger age, a poorer baseline lung function, and a history of multiple previous pulmonary exacerbations were risk factors for pulmonary exacerbations. This study was done in adults and a few adolescents, and all patients had chronic, multidrug-resistant bacterial infections. In our study, chronic MRSA and/or PA and/or $B C C$ infection was a risk factor for pulmonary exacerbation. Chronic MRSA infection has been the subject of several studies. Some demonstrated that it impaired lung function and required more intravenous antibiotic courses. ${ }^{17}$ In our study, it was observed that it increased the possibility for pulmonary exacerbations. In 2008, Jarad et al., ${ }^{18}$ showed that PA infection, a poor lung function, and CFRD were risk factors for pulmonary exacerbations in the adult population. Chronic $\mathrm{PA}^{19,20}$ and $\mathrm{BCC}^{21}$ infection was associated with a progressive deterioration of lung function; in this study, a chronic infection with any of these bacteria was a major risk factor for an exacerbation in children with $\mathrm{CF}$.

The association between nutritional status and lung function in CF is well-known. ${ }^{22}$ Patients with a better nutritional status have better lung

TABle 3. Poisson predictive logistic regression model using pulmonary exacerbations as a dependent outcome measure

\begin{tabular}{lcc}
\hline & RR $(95 \%$ CI) & $p$ \\
\hline Lower BMI Z-score & $1.45(1.13,1.88)$ & 0.002 \\
p.F508del mutation & $3.23(1.01,10.58)$ & 0.050 \\
BCC & $3.69(1.61,8.48)$ & 0.002 \\
PA & $1.89(1.11,3.20)$ & 0.017 \\
MRSA & $2.32(1.35,3.99)$ & 0.002 \\
\hline
\end{tabular}

BMI: body mass index; BCC: Burkholderia cepacia complex; PA: Pseudomonas aeruginosa; MRSA: methicillin-resistant Staphylococcus aureus; RR: relative risk;

$\mathrm{CI}$ : confidence interval. function values and a longer survival. In our study, a poor nutritional status was a risk factor for pulmonary exacerbation.

p.F508del is the most common mutation in the worldwide population. In Argentina, in $59 \%$ of cases, it is in at least one of the alleles of CF patients. ${ }^{23}$ It is a serious mutation and, in our study, it was shown to be a risk factor for pulmonary exacerbation.

Like in the study by Sanders, ${ }^{12}$ a fourth of patients in our study failed to recover their lung function. In 2017, Sanders ${ }^{24}$ demonstrated that failure to recover lung function was associated with a shorter time until the next exacerbation, a poor recovery from the next exacerbation, and a greater possibility of future exacerbations. The same author also demonstrated that a higher number of exacerbations was related to an increased rate of lung function deterioration. ${ }^{9}$

Our study has two limitations. One is that $\mathrm{FEV}_{1}$ was measured at the end of hospitalization to check if patients had recovered their baseline lung function. The study by Sanders ${ }^{12}$ suggests that the best moment to assess the recovery of lung function is in the months after an exacerbation. The second limitation is its retrospective nature and 12-month duration period. In the future, it would be advisable to plan a prospective, multicenter study with a longer study period. It is worth noting that this study was done in the pediatric population and,

FIGURE 2. Baseline forced expiratory volume in the first second (3-6 months before exacerbation) and at the end of exacerbation as a percentage of that predicted in patients with exacerbation

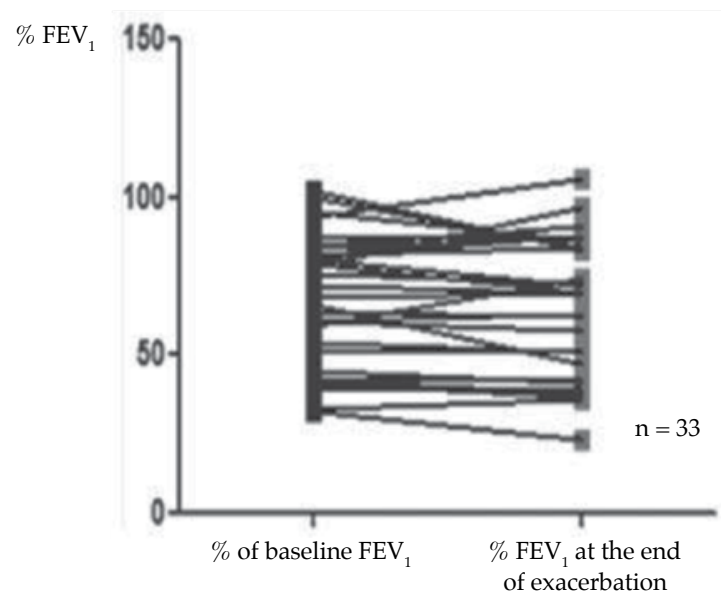

$\mathrm{FEV}_{1}$ : forced expiratory volume in the first second. 
to date, there are no studies on the risk factors for pulmonary exacerbation in this age group.

A pulmonary exacerbation is a critical event in the determination of pulmonary disease course in CF. Knowing the risk factors associated with pulmonary exacerbations may help to more strictly monitor patients with said factors and thus prevent them.

\section{CONCLUSIONS}

The presence of the p.F508del mutation, a poor nutritional status, and chronic MRSA, PA and $\mathrm{BCC}$ colonization are predictive risk factors for pulmonary exacerbation in pediatric patients with CF. After a pulmonary exacerbation, a fourth of patients failed to recover their lung function.

\section{REFERENCES}

1. Bhatt J. Pulmonary exacerbation in Cystic Fibrosis. Eur Respir Rev. 2013; 22(129):205-16.

2. Flume PA, Mogayzel PJ Jr, Robinson KA, Goss CH, et al. Cystic fibrosis pulmonary guidelines: treatment of pulmonary exacerbations. Am J Respir Crit Care Med. 2009; 180(9):802-8.

3. Cystic Fibrosis Foundation Patient Registry. 2016 Patient Registry Annual Data Report. Bethesda, Maryland: Cystic Fibrosis Foundation; 2016. [Accessed on: March 13 ${ }^{\text {th }}, 2019$ ]. Available at: https: / / www.cff.org/Research/ResearcherResources / Patient-Registry/2016-Patient-Registry-Annual-Data-Report/.

4. Rosenfeld M, Emerson J, Williams-Warren J, Pepe M, et al. Defining a pulmonary exacerbation in cystic fibrosis. $J$ Pediatr. 2001; 139(3):359-65.

5. Bilton D, Canny G, Conway S, Dumcius S, et al. Pulmonary exacerbation: Towards a definition for use in clinical trials. Report from the Euro Care CF Working Group on outcome parameters in clinical trials. J Cyst Fibros. 2011; 10(Suppl 2):S79-81.

6. Ramsey BW, Pepe MS, Quan JM, Otto KL, et al. Intermittent administration of inhaled tobramycin in patients with cystic fibrosis. Cystic Fibrosis Inhaled Tobramycin Study Group. N Engl J Med. 1999; 340(1):23-30.

7. Saiman L, Marshall BC, Mayer-Hamblett N, Burns JL, et al. Azithromycin in patients with cystic fibrosis chronically infected with Pseudomonas aeruginosa: a randomized controlled trial. JAMA. 2003; 290(13):1749-56.

8. Fuchs HJ, Borowitz DS, Christiansen DH, Morris EM, et al. Effect of aerosolized recombinant human DNase on exacerbations of respiratory symptoms and on pulmonary function in patients with cystic fibrosis. The Pulmozyme Study Group. N Engl J Med. 1994; 331(10):637-42.
9. Sanders DB, Bittner RC, Rosenfeld M, Redding GJ, et al. Pulmonary exacerbations are associated with subsequent $\mathrm{FEV}_{1}$ decline in both adults and children with cystic fibrosis. Pediatr Pulmonol. 2011; 46(4):393-400.

10. Britto M, Kotagal U, Hornung R, Atherton HD, et al. Impact of recent pulmonary exacerbations on quality of life in patients with cystic fibrosis. Chest. 2002; 121(1):64-72.

11. De Boer K, Vandemheen KL, Tullis E, Doucette S, et al. Exacerbation frequency and clinical outcomes in adult patients with cystic fibrosis. Thorax. 2011; 66(8):680-5.

12. Sanders D, Bittner R, Rosenfeld M, Hoffman L, et al. Failure to recover to baseline pulmonary function after cystic fibrosis pulmonary exacerbation. Am J Respir Crit Care Med. 2010; 182(5):627-32.

13. Schechter MS, Shelton BJ, Margolis PA, Fitzsimmons SC. The association of socioeconomic status with outcomes in cystic fibrosis patients in the United States. Am J Respir Crit Care Med. 2001; 163(6):1331-7.

14. Block J, Vandemheen K, Tullis E, Fergusson D, et al, Predictors of pulmonary exacerbations in patients with cystic fibrosis infected with multi-resistant bacteria. Thorax. 2006; 61(11):969-74.

15. Lee TW, Brownlee KG, Conway SP, Denton M, et al. Evaluation of a new definition for chronic Pseudomonas aeruginosa infection in cystic fibrosis patients. J Cyst Fibros. 2003; 2(1):29-34.

16. Liou TG, Adler FR, Fitsimmons SC, Cahill BC, et al. Predictive 5-year survivorship model of cystic fibrosis. Am J Epidemiol. 2001; 153(4):345-52.

17. Sawicki MD, Rasouliyan L, Pasta DJ, Regelmann WE, et al. The impact of incident methicillin resistant staphylococcus aureus detection on pulmonary function in cystic fibrosis. Pediatr Pulmonol. 2008; 43(11):1117-23.

18. Jarad NA, Giles K. Risk factors for increased need for intravenous antibiotics for pulmonary exacerbations in adult patients with cystic fibrosis. Chron Respir Dis. 2008; 5(1):29-33.

19. Kosorok M, Zeng L, West S, Rock M, et al. Acceleration of lung disease in children with cystic fibrosis after Pseudomonas aeruginosa acquisition. Pediatr Pulmonol. 2001; 32(4):277-87.

20. Demko C, Byard P, Davis P. Gender differences in cystic fibrosis: Pseudomonas aeruginosa infection. J Clin Epidemiol. 1995; 48(8):1041-9.

21. Waters V. New treatments for emerging cystic fibrosis pathogens other than Pseudomonas. Curr Pharm Des. 2012; 18(5):696-725.

22. Konstan MW, ButlerSM, WohlME,Stoddard M, etal.Growth and nutritional indexes in early life predict pulmonary function in cystic fibrosis. J Pediatr. 2003; 142(6):624-30.

23. Visich A, Zielenski J, Castaños C, Diez G, et al. Complete screening of the CFTR gene in Argentine cystic fibrosis patients. Clin Genet. 2002; 61(3):207-13.

24. Sanders DB, Qianqian Z, Li Z, Farrell PM. Poor recovery for cystic fibrosis pulmonary exacerbations is associated with poor long-term outcomes. Pediatr Pulmonol. 2017; 52(10):1268-75. 